\title{
Experimental Organism Mixed Lymphoma Neoplasm
}

National Cancer Institute

\section{Source}

National Cancer Institute. Experimental Organism Mixed Lymphoma Neoplasm. NCI

Thesaurus. Code C114111.

A malignant neoplasm composed of a mixed lymphocyte population. 